\title{
Neutrophils: driving progression and poor prognosis in hepatocellular carcinoma?
}

Jane Margetts ${ }^{1,2,10}$, Laura F Ogle ${ }^{2,10}$, Stephen L Chan ${ }^{3,4}$, Anthony W H Chan ${ }^{5}, \mathrm{~K}$ C Allen Chan ${ }^{6}$, David Jamieson ${ }^{2}$, Catherine E Willoughby ${ }^{2}$, Derek A Mann ${ }^{7}$, Caroline L Wilson ${ }^{7}$, Derek M Manas ${ }^{8}$, Winnie Yeo ${ }^{3}$ and Helen L Reeves ${ }^{\star 1,9}$

${ }^{1}$ Northern Centre for Cancer Care, Newcastle-upon-Tyne Hospitals NHS Foundation Trust, Newcastle upon Tyne NE2 7DN, UK; ${ }^{2}$ Northern Institute for Cancer Research, The Medical School, Newcastle University, Paul O'Gorman Building, Framlington Place, Newcastle-upon-Tyne NE2 4HH, UK; ${ }^{3}$ State Key Laboratory in Oncology of South China, Department of Clinical Oncology, Chinese University of Hong Kong, Hong Kong, Hong Kong; ${ }^{4}$ Institute of Digestive Disease, Chinese University of Hong Kong, Hong Kong, Hong Kong; ${ }^{5}$ State Key Laboratory in Oncology of South China, Department of Anatomical and Cellular Pathology, Chinese University of Hong Kong, Hong Kong, Hong Kong; 'S State Key Laboratory in Oncology of South China, Department of Chemical Pathology, Chinese University of Hong Kong, Hong Kong, Hong Kong; ${ }^{7}$ Institute of Cellular Medicine, The Medical School, Newcastle University, Framlington Place, Newcastle-upon-Tyne, NE2 4HH, UK; ${ }^{8}$ Hepatopancreatobiliary Unit, Surgical Directorate, Freeman Hospital, Newcastle upon Tyne Hospitals NHS Foundation Trust, Newcastle upon Tyne NE2 7DN, UK and ${ }^{9}$ The Liver Unit, Freeman Hospital, Newcastle upon Tyne Hospitals NHS Foundation Trust, Newcastle upon Tyne NE2 7DN, UK

Background: Irrespective of the underlying aetiology, $90 \%$ of hepatocellular carcinomas arise and progress on a background of chronic inflammation. We have explored the independent prognostic value of circulating inflammatory cells.

Methods: Peripheral blood count data sets from 583 consecutive patients presenting to a single UK centre (2000-2010) were analysed for associations with tumour stage, liver function, performance status (PST) and survival. Validation was in an independent Hong Kong cohort (585 patients; 2007-2013).

Results: In both UK and Hong Kong cohorts, neutrophils, platelets, lymphocytes, the neutrophil/lymphocyte ratio (NLR) and the Systemic Immune-Inflammation Index (SII) correlated stepwise, either increasing or decreasing (lymphocytes), with tumour node metastasis (TNM) and Childs-Pugh stage, PST and consequently with the combined Barcelona Clinic for Liver Cancer stage. Survival analyses confirmed the NLR and SII as highly significant prognostic biomarkers. Focused on individual cell types, only the neutrophil count was independently associated with both TNM stage and PST, as well as being significantly and independently associated with poorer survival.

Conclusions: In this study of 1168 patients, neutrophils alone, rather than lymphocytes or platelets, were independently associated with outcome. These data support further characterisation of a potentially distinctive role for neutrophils as facilitators of tumour progression and deteriorating performance.

Hepatocellular carcinoma (HCC) has the second highest cancerrelated mortality worldwide (International Agency for Research on Cancer, 2014), reflecting its rising incidence, late-stage presentation and limited therapeutic options. Globally, HCC most commonly arises in individuals with underlying chronic liver diseases (CLDs) caused by hepatitis B virus (HBV) or hepatitis C

\footnotetext{
*Correspondence: Dr HL Reeves; E-mail: helen.reeves@ncl.ac.uk

${ }^{10}$ These authors contributed equally to this work.
}

Received 1 July 2017; revised 4 September 2017; accepted 9 October 2017; published online 9 November 2017

(C) 2018 Cancer Research UK. All rights reserved 0007-0920/18 
virus. In the $\mathrm{UK}$, where the prevalence of these infections is relatively low, HCC was the ninth commonest cause of cancer mortality in 2014, accounting for 5091 deaths (Cancer Research UK, 2017). Of growing concern, although deaths from other cancers are falling in the UK, HCC-related mortality is rising, having doubled over the last decade. The reasons include a worrying rise in alcohol-related liver disease (ARLD) (Williams et al, 2014), but also a dramatic increase in the prevalence of obesity and type 2 diabetes mellitus-related non-alcoholic fatty liver (NAFLD) (Dyson et al, 2014). Driven by stage, potentially curative treatments (resection, transplantation and ablation) are offered to $<15 \%$ of patients with HCC. Although first-line palliative locoregional therapy and the multikinase inhibitor sorafenib (Llovet et al, 2008) are increasingly used, owing to impaired liver function or deteriorating performance status (PST), up to $50 \%$ of those affected are offered supportive care only and overall survival is poor (Dyson et al, 2014). The recent failures of targeted second line agents in phase III trials offer limited hope for change, the lack of progress being attributed to a failure to identify the key drivers of hepatocarcinogenesis, as well as the lack of biomarkers guiding stratification of therapy (Llovet et al, 2016).

Molecular aberrations in the tumour cells are undoubtedly oncogenic drivers in HCC (Hoshida et al, 2009; Nault et al, 2013; Schulze et al, 2015; Villanueva et al, 2015; Zucman-Rossi et al, 2015), but interactions with inflammatory cells within the tumour microenvironment may also be key (Hanahan and Weinberg, 2011). Inflammation is a cardinal feature of progressive liver disease and predisposition to HCC, the latter attributed in part to inflammatory cell generated oxidative stress and DNA damage. In addition, however, an inflammatory response associated with a cancer can either aid its elimination or drive its progression. The presence of tumour-infiltrating lymphocytes in HCC resections is associated with better survival (Chew et al, 2010), although cumulative evidence from murine and human studies recognises a balance between the pro-tumour $v s$ antitumour effects of different lymphocyte subsets, including regulatory CD4 and cytotoxic CD8 lymphocytes (Gooden et al, 2011). Similarly, for other immune cells, such as macrophages and neutrophils, recognition of protumour and anti-tumour subtypes (Hernandez-Gea et al, 2013) and the role of platelets is growing (Carr and Guerra, 2013). The present therapeutic focus is on immune checkpoint inhibitors, a class of drugs which promotes the CD8 anti-tumour response. Although Phase III studies in patients with HCC are awaited, data from early phase trials suggests that this approach may offer a significant survival advantage for some patients (Kudo, 2015). How to identify patients that will respond to checkpoint inhibition is as yet unknown and the lack of routine diagnostic biopsy for HCC owing to good diagnostic performance of contrast enhanced cross sectional imaging combined with the small risks of haemorrhage and tumour seeding in patients with cirrhosis (Sherman and Bruix, 2015) - hampers the development of clinically relevant biomarkers (Torbenson and Schirmacher, 2015) for these and other therapies. Monitoring tumour responses can also be challenging.

Given the difficulties acquiring liver tissues, circulating biomarkers that accurately reflect the tumour microenvironment are needed and in parallel to the research focused on the localised tumour immune response, a number of studies exploring circulating inflammatory biomarkers - which potentially have prognostic or clinically relevant predictive value - have been reported in patients with HCC. Largely restricted to smaller studies in treatment subgroups, most often in patients with underlying viral hepatitis, an elevated neutrophil to lymphocyte ratio (NLR), reflecting either increases in neutrophils or reduced lymphocytes (Xu et al, 2014), reportedly has prognostic value post resection (Gomez et al, 2008), liver transplantation (Limaye et al, 2013) and ablation (Dan et al, 2013). Recently the Systemic ImmuneInflammation Index (SII) score - based on numbers of neutrophils, lymphocytes and platelets ( $\mathrm{Hu}$ et al, 2014) - has been proposed as an alternative.

We have interrogated peripheral inflammatory cell counts in two large patient cohorts (583 and 585 patients), wanting to explore the relative biological contributions of neutrophils, lymphocytes and platelets in a more comprehensive fashion across all HCC stages, underlying aetiologies and treatment subsets. Searching for driving influences, our aim was to further understand their associations with HCC progression.

\section{MATERIALS AND METHODS}

Patient cohorts. We have assessed standard blood count data in two patient cohorts. Cohort 1 included patients previously described (Dyson et al, 2014). Briefly, these were consecutive patients managed by the Newcastle HCC multidisciplinary meeting between the years 2000 and 2010, guided by internationally accepted guidelines (Bruix et al, 2001), including the Barcelona Clinic for Liver Cancer (BCLC) staging algorithm (Llovet et al, 2004; Bruix and Sherman, 2005). Tumour node metastasis (TNM), Childs-Pugh (CP) and BCLC stages were documented, as were demographic and clinical parameters, Eastern Cooperative Oncology Group (ECOG) PST, treatments and survival. The study was registered with the hospital trust audit department and cases were followed until 31 March 2017, defining a minimum period of 6 years post diagnosis. Blood cell counts, including platelets, neutrophils and lymphocyte data at the time of diagnosis and staging were obtained retrospectively from patients' electronic records. Of 632 consecutive patients with HCC, 49 cases were excluded as complete full blood count data were unavailable, leaving 583 included Newcastle patients. The SII index was calculated as SII $=P \times N / L$, where $P, N$ and $L$ were the peripheral platelet, neutrophil and lymphocyte counts, respectively ( $\mathrm{Hu}$ et al, 2014).

The Newcastle patient data set was used as the exploratory cohort, investigating relationships between circulating immune cells and scores with clinical parameters and outcomes. A second cohort of consecutive HCC patients $(n=585)$ was used for validation purposes. The cohort consisted of HCC patients presenting to the Prince of Wales Hospital in Hong Kong between 1 July 2007 and 31 July 2013 (Chan et al, 2012, 2017), also followed until 31 March 2017. The construction of this cohort was approved by the Joint Chinese University of Hong Kong - New Territories East Cluster Research Ethics Committee.

Statistical analyses. All statistical analyses were done with SPSS for Windows, version 22 (SPSS Inc., Chicago, IL, USA), licensed to Newcastle University. Figures were drawn using GraphPad Prism 6 (GraphPad Inc., La Jolla, CA, USA). Differences between categorical variables were assessed by Pearson's $\chi^{2}$-test. Differences between groups of continuous variables that were non-parametrically distributed were assessed using Mann-Whitney or Kruskal-Wallis tests. Bivariate correlations were evaluated using Spearman's rank correlation coefficient. For categorical data, multinomial regression was used to assess the independent associations of multiple variables. A $P$-value of $<0.05$ was considered significant. Survival was recorded as months from diagnosis to 31 March 2017. Differences in cumulative survival were determined using the Kaplan-Meier method and a log-rank test. The Cox proportional hazards regression model was used to identify parameters associated with survival. Factors initially considered by univariate analysis included age, gender, associated aetiology, presence cirrhosis, number of tumours, size of largest tumour, presence of extrahepatic disease or portal vein invasion/ thrombosis, presence of ascites or encephalopathy, serum $\alpha$-fetoprotein (AFP), serum albumin, serum bilirubin, prothrombin 
time, whole blood count platelets, neutrophils, lymphocytes, as well as the presence of constitutional symptoms. A cutoff of $P<0.05$ was used to select variables entered into the multivariate model.

\section{RESULTS}

Differences and similarities between the Newcastle and Hong Kong cohorts. The demographics of the Newcastle and Hong Kong cohorts of patients with HCC are compared and contrasted in Table 1, alongside the combined cohort of 1168 patients. Similar numbers of patients were studied, but in Newcastle these reflected patients presenting over an 11 year period, compared with 5.5 years in Hong Kong. The Newcastle cohort median age was significantly greater, at 69 vs 60 years, and included more women. In addition, there were striking differences in underlying liver disease aetiology, with the majority of HCC arising on a background of fatty liver disease in the Newcastle cohort, vs HBV in the Hong Kong cohort. A greater proportion of Newcastle patients had no evidence of underlying CLD of any kind. Despite this, a greater number of Hong Kong patients developed HCC in the absence of cirrhosis - as is commonly recognised in the presence of chronic HBV infection. Notably, the average tumour size and TNM stage were more advanced in the Hong Kong cohort. While a greater proportion in Hong Kong had extrahepatic disease and portal vein invasion, which contribute to stage BCLC $\mathrm{C} / \mathrm{D}$ classifications - a greater proportion of Newcastle patients were classed as BCLC stage D, reflecting more Newcastle cases staged as ECOG PST 3 or 4 and/or liver function CP C. Peripheral blood cell count data is also summarised in Table 1. Although there were no differences in mean neutrophil or platelet counts, the mean lymphocyte count in the Hong Kong Cohort was slightly but significantly lower than the Newcastle cohort, with consequently higher NLR and SII. The primary surgical treatment in Newcastle was orthotopic liver transplantation, whereas in Hong Kong Prince of Wales Hospital it was resection. Newcastle patients with intermediate stage disease were more likely to receive loco-regional therapy, compared with Sorafenib in the Hong Kong cohort reflecting the greater numbers in Hong Kong with extrahepatic disease/TNM IV at presentation, but also the temporal differences in the cohorts and the availability of Sorafenib at the time of patient presentation.

Consequent to the mostly advanced stages at presentation as well as limited therapeutic options as previously described, irrespective of the centre and cohort differences, very similar numbers of patients $(41 \%$ and $38 \%$, respectively) received supportive care only. Despite the differences between the cohorts, this similarity was possibly the most telling, as there was no significant survival difference between the two. Median survival in the Newcastle cohort was 11.9 months compared with 8.4 months in Hong Kong (Kaplan-Meier, $P=0.219$ ).

Neutrophils and platelets rise with advanced tumour stage, whereas lymphocytes fall. In both the Newcastle and Hong Kong cohorts, the NLR and the SII rose in association with advancing TNM, CP and PST, as well as with the BCLC stage, which combines the three. Focusing on the different cell types rather than combination scores, significant incremental increases in median neutrophil count paralleled increases in TNM and PST. Conversely, lymphocytes tended to fall with advancing stages. Platelets fell significantly with advancing CP stage, but increased in association with TNM and BCLC stage. Figures for Newcastle and Hong Kong cohorts independently are shown in Supplementary Figures 1-4, whereas the combined cohort data summarising changes with TNM, CP and PST is presented in Figures 1-3.
As each of these cell counts can decline in patients with cirrhosis and portal hypertension, which are both drivers of advancing $\mathrm{CP}$ and BCLC stage, cirrhosis as a potential confounder was considered. Each of the cell counts, platelets most notably, correlated negatively with the presence of cirrhosis (Spearman's bivariate correlations: neutrophils $-0.244, P<0.001$; lymphocytes $-0.093, P=0.001$; platelets $-0.426, P<0.001)$. Restricting the assessment of associations of the cell types with the different staging systems to patients without cirrhosis (403 patients), the patterns of change and their significance were similar to those described above (data not shown). The only difference was a lack of a significant association between platelets and CP stage.

The stepwise increases in median neutrophil counts with PST as shown in Figure 3 were interesting and although hard to dissociate from advancing cancer stage, the relationship between neutrophils and PST persisted $(P<0.001)$ in a multinomial regression analysis, which included other factors significantly associated with PST in a univariate analysis (peripheral lymphocyte and platelet counts, but also age, sex, aetiology, cirrhosis, tumour number, tumour size, vascular invasion, extrahepatic disease, ascites, prothrombin time, serum albumin, bilirubin, AFP and Newcastle vs Hong Kong cohorts). Data are shown in Supplementary Table 1, where variables at each ECOG PST stage were compared with the last and most advanced stage (Stages 3 and 4 combined). Although age, tumour factors (size, vascular invasion and extrahepatic disease) and liver function features (ascites) were significantly associated compared with ECOG PST 0, only falling serum albumin and rising neutrophil count were incrementally and significantly associated at each stage ECOG PST stage. Neither lymphocyte nor platelet count associations were independently significant.

The prognostic value of the NLR. A number of previous studies have explored the NLR in patients with HCC. An NLR of $\geqslant 5$ vs $<5$ was shown to be a poor prognostic indicator in a cohort of 96 patients undergoing resection (Gomez et al, 2008), as well as in a cohort of 160 patients treated with liver transplantation (Limaye et al, 2013). This cutoff value in fact performed poorly in our combined cohort of 152 surgically treated patients, where only 20 had an NLR $\geqslant 5$. Using a median cutoff value for our combined surgical cohort of $\geqslant 2.5$ vs $<2.5$ (Newcastle cases 41 vs 43; Hong Kong cases 35 vs 33), there was again no significant difference in either median or long term survival between the groups. The majority of patients undergoing surgical treatment in our cohorts (87\%) had HCC classed as TNM stage I or II, without an obvious difference in NLR between these stages of disease (Figure 1).

In patients with all stages of disease, however, the whole cohort median NLR value of 3.15 was a very highly significant discriminator by Kaplan-Meier analysis. Median survival in the combined cohort $(n=578<3.15$; vs $n=588 \geqslant 3.15)$ was 18.8 months vs 4.5 months $(P<0.001$, Kaplan-Meier, as shown in Figure 4). In a multivariate Cox regression analysis, including other variables significant in a univariate analysis (tumour number, largest diameter, prothrombin time, albumin, bilirubin and AFP as continuous variables; the presence of ascites, portal vein invasion, extrahepatic disease, constitutional symptoms, underlying aetiology and primary treatment group as categorical variables), an NLR $\geqslant 3.15$ was independently associated with poorer survival (hazard ratio (HR) $0.71 ; 0.62-0.81 ; P<0.001$ ).

The prognostic value of the SII. Recognising the potential contribution of circulating platelets, with a particular interest in the role they play in promoting circulating tumour cell survival and metastases, Hu et al (2014) proposed the SII index as an alternative and possibly more informative biomarker. To calculate the SII, the NLR is multiplied by the platelet count. Hu et al (2014) used a retrospective cohort of 133 resection patients to propose an SII $>330 \times 10^{9}$ as having poor prognostic value, which they validated 
Table 1. Patient cohorts

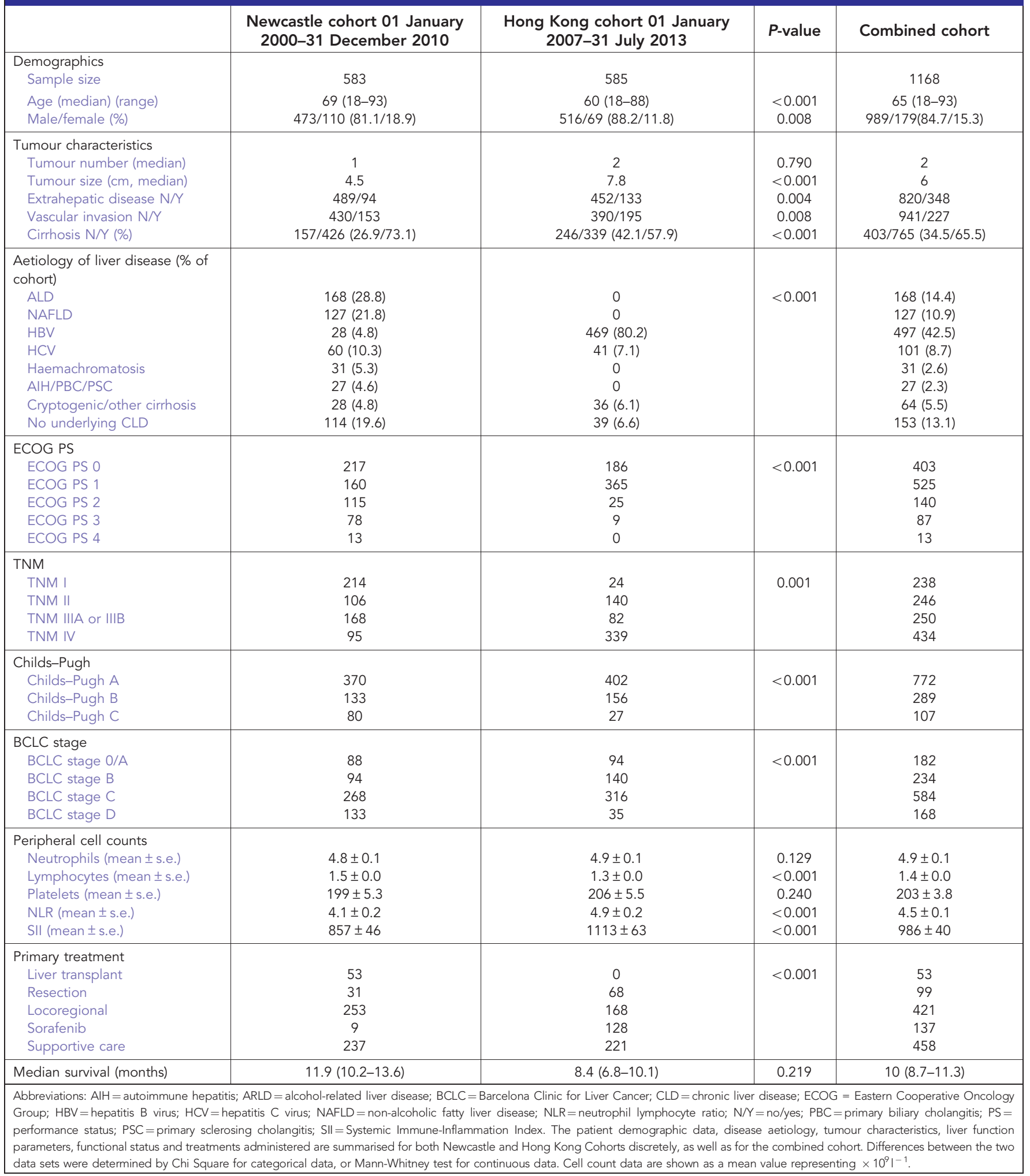

in a prospective cohort of 123 patients. An SII $>300$ showed promise in our small transplanted cohort $(n=53)$, trending towards significance (median survival 90.8 months $(n=31)$, vs 72.2 months $(n=22)$, for SII $<300$ or $>300$, respectively, $P=0.280)$. In our combined resection series $(n=99)$, however, the trend was reversed (median survival 82.4 months $(n=33)$ vs 125.0 months $(n=66) ; P=0.164)$. For these small groups of surgically treated patients, therefore, we did not confirm a prognostic value in terms of survival. In our entire combined cohort, however, an SII > 300 was present in 847 of 1168 patients, associated with a median survival of 6.9 months compared with 21.9 months for those with an SII $<300(P<0.001$, KaplanMeier). The survival curves comparing patients below and above the median SII of 569 ( $n=582$ vs 584) are shown in Figure 4 (18.9 months vs 4.4 months, $P<0.001)$. In a multivariate Cox regression analysis similar to that described for the NLR, a median SII above 

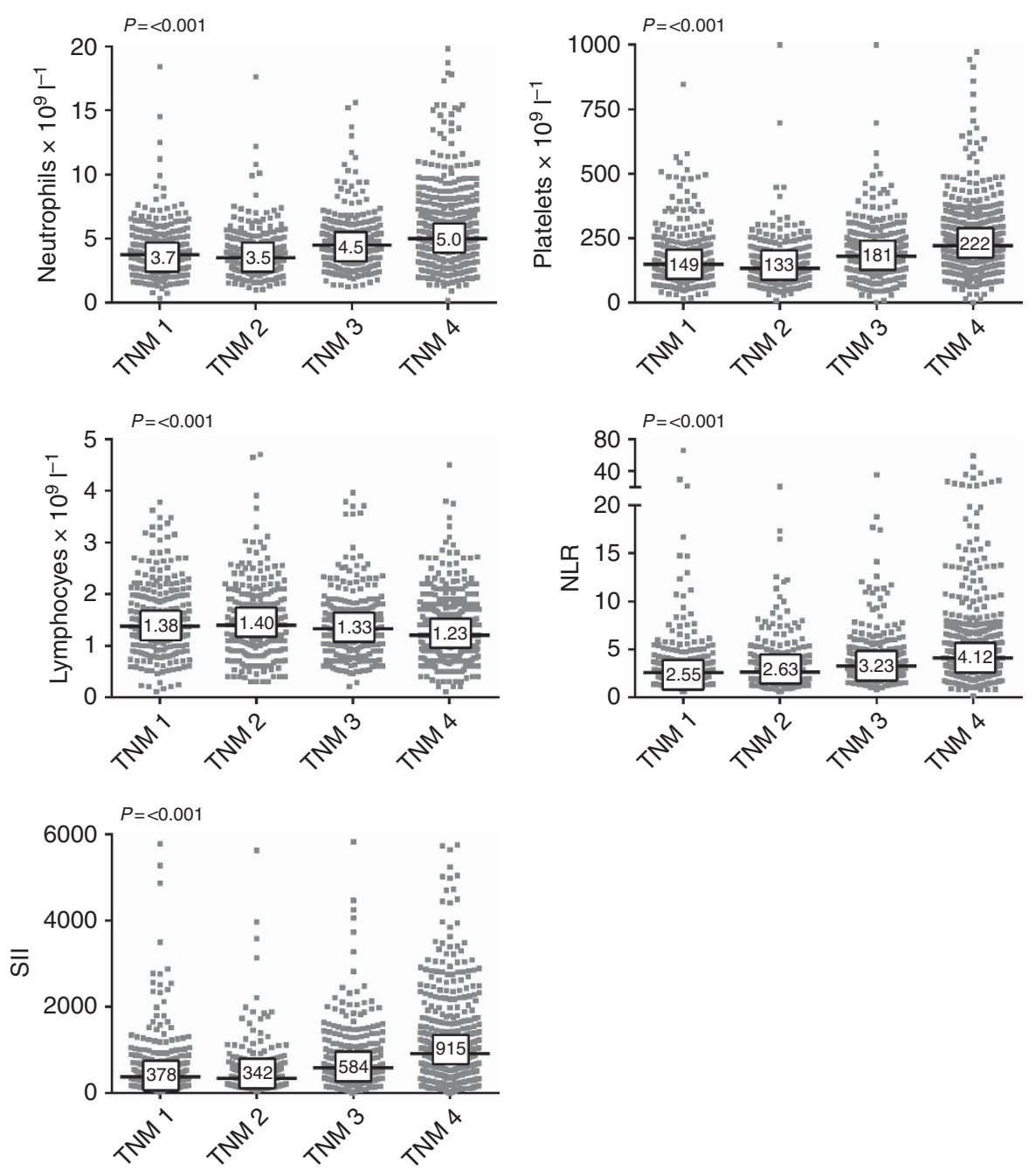

Figure 1. Inflammatory cells associations with TNM stage. Newcastle and Hong Kong data sets combined associations with advancing tumour node metastasis (TNM) stage for neutrophil, platelets and lymphocytes, as well as NLR (neutrophil to lymphocyte ratio) and SII (Systemic ImmuneInflammation Index) are shown. Statistical differences were assessed by Kruskal-Wallis test.

the median value was independently associated with poorer survival (HR 0.70; 0.60-0.81, P<0.001).

In our combined cohort, both the NLR and the SII as continuous variables correlated highly significantly with tumour number, tumour size, vascular invasion, AFP and extrahepatic disease. The stronger correlations were with the SII, in particular with tumour size $(0.498$, Spearman's correlation, $P<0.001)$.

Exploration of the contributions of distinct inflammatory cells. In the large combined cohort studied, including all stages of disease and underlying aetiologies, we aimed to explore the independent contributions and associations of the cell types, rather than the combination scores. Focused on survival, univariate analyses for the Newcastle cohort, the Hong Kong cohort and the combined cohort, followed by multivariate Cox regression analysis including those variables with a $P$-value $<0.05$, are shown in Table 2. Variables independently significantly associated with adverse survival in the Newcastle cohort, validated in the Hong Kong cohort, included tumour factors (increased HCC number or size and the presence of vascular invasion or extrahepatic disease), falling serum albumin, rising bilirubin, the presence of constitutional symptoms and a rising peripheral neutrophil count. Additional factors in the Hong Kong cohort significantly associated with adverse survival included the presence of ascites, an elevated AFP, an elevated prothrombin time, as well as a lower lymphocyte count. In the larger combined cohort, where there was no significant difference in survival between the two cohorts, independent significance persisted for serum AFP and the presence of ascites, but not for the prothrombin time or lymphocyte count. Notably, in both cohorts, the peripheral neutrophil count was the only inflammatory cell count with independent prognostic value in a multivariate Cox regression analysis including all variables with a $P$-value $<0.05$. Treated as a categorical variable, taking the neutrophil median value as the cutoff discriminating two groups of patients $(<4.2 v s \geqslant 4.2)$, the difference in median survival was 17.3 vs 5.1 months (Kaplan-Meier; $P<0.001$, Figure 4), with similarly independent significance (HR $0.77,0.66-0.89 ; P=0.001$ ). The survival curves for both lymphocytes and platelet counts, above and below their median values, were significant by Kaplan-Meier analyses, also shown in Figure 4. Again, neither was significant in a multivariate analysis.

Exploring neutrophil associations with the other inflammatory cells in the combined cohort, a positive and highly significant correlation was noted with platelets $(0.565, P<0.001)$. A much weaker but still significant correlation was also observed with lymphocytes $(0.108, P<0.001)$. As described, the presence or absence of cirrhosis had a significant impact on cell counts, with median values (SI units $\times 10^{9} 1^{-1}$ ) in the absence and presence of cirrhosis being 5.0 vs 3.9; 246 vs 143; 1.6 vs 1.4 for neutrophils, platelets and lymphocytes, respectively $(P<0.001 ; P<0.001$ and 

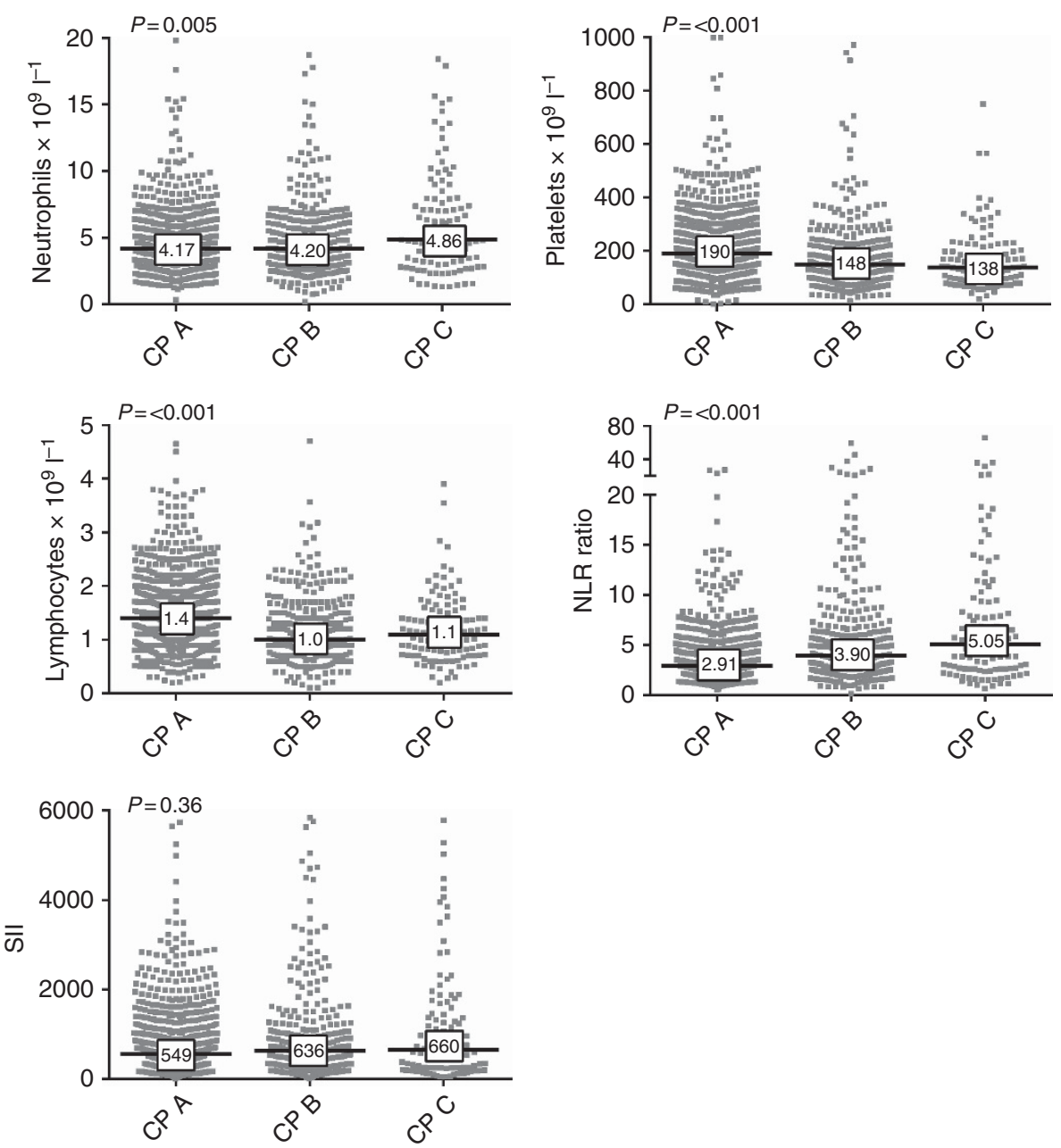

Figure 2. Inflammatory cells associations with CP stage. Newcastle and Hong Kong data sets combined associations with advancing Childs-Pugh (CP) stage for neutrophil, platelets and lymphocytes, as well as NLR (neutrophil to lymphocyte ratio) and SII (systemic immune index) are shown. Statistical differences were assessed by Kruskal-Wallis test.

$P=0.001$ respectively, Mann-Whitney). Focused only on patients without cirrhosis, the highly significant correlation between neutrophils and platelets remained (Spearman's 0.402, $P<0.001$ ), whereas the weak association between neutrophils and lymphocytes was lost (Spearman 0.012, $P=0.805$ ). Revisiting survival, restricting analyses to patients without cirrhosis, neutrophils remained the only circulating cell significantly and independently associated with survival (data not shown).

\section{DISCUSSION}

Studying two large cohorts of patients with HCC from Newcastle and Hong Kong has provided the opportunity to explore the prognostic value of peripheral cell counts and combination scores in much greater depth than before, as previous case series have tended to be small and/or focused on specific treatment groups. Including all patients presenting to our units over defined periods of time has captured all stages of disease. Furthermore, the quite marked differences between the Newcastle and Hong Kong cohorts has facilitated a comprehensive assessment of the value of combined scores, as well as the contributions of individual cell types, which goes beyond the influence of important factors such as age, sex, underlying aetiology of disease, geographical location, presence or absence of cirrhosis and treatments received.

Circulating inflammatory cell counts and combined inflammatory scores generally increased with more advanced TNM, CP and
BCLC stage, with the exception being lymphocytes, which were inversely related. Notably, lymphocyte counts were slightly lower in the Hong Kong cohort independently of stage and variance in the systemic inflammatory response associated with geographical location have been previously reported (Dolan et al, 2017). As cirrhosis with portal hypertension has a very well established impact on circulating cell counts, it was important to confirm that the associations with advancing stage persisted even in the absence of cirrhosis, supporting the influence of the advancing cancer on these peripheral cell counts. The association of neutrophils with PST was notable and persisted in a multinomial regression analysis independently of other highly significantly associated factors. This raises the possibility that elevated numbers of circulating neutrophils may contribute to the cancer related cachexia syndrome, of which declining PST is a critical part. Although not well understood, an inflammatory tumour microenvironment has a key role in the development of cancer related cachexia, with neutrophil infiltration in association with elevated levels of circulating neutrophils reported in animal studies (Chiba et al, 2014). Not only does PST have a major impact on cancer patients' survival, it also impairs the capacity to withstand the anti-cancer treatments that might prolong survival.

Turning to prognosis, in this study we have comprehensively assessed the prognostic survival value of both the NLR and the SII. Taken across all stages of disease in these large cohorts, rising values undoubtedly have prognostic value, although their clinical value within stages of disease and treatment categories remains 

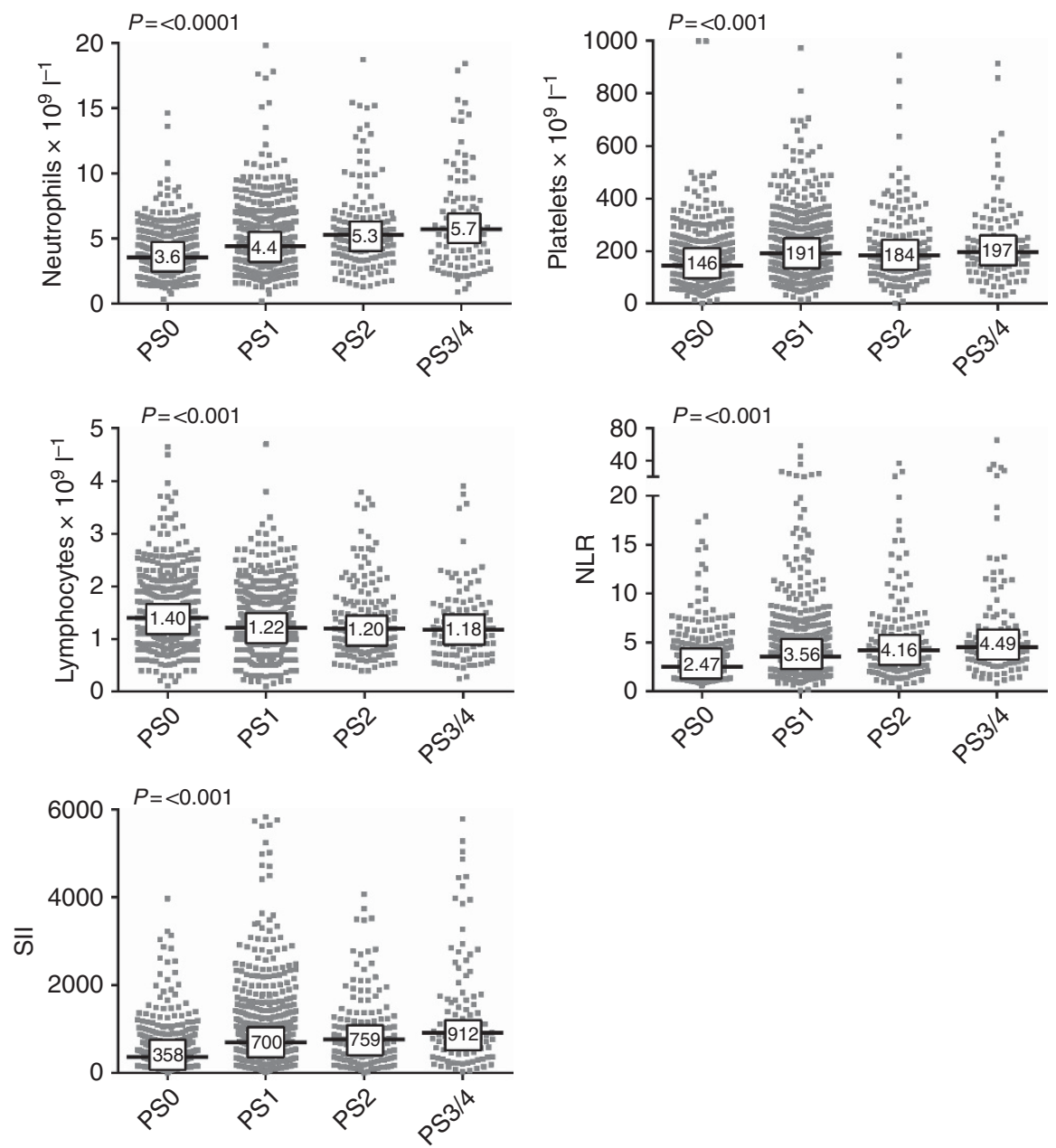

Figure 3. Inflammatory cells associations with PST. Newcastle and Hong Kong data sets combined associations with advancing Eastern Cooperative Oncology Group (ECOG) performance status (PST) for neutrophil, platelets and lymphocytes, as well as NLR (neutrophil to lymphocyte ratio) and SII (Systemic Immune-Inflammation Index) are shown. Statistical differences were assessed by Kruskal-Wallis test.

uncertain. In our combined series of 152 surgically treated patients, where the majority in our series (87\%) were TNM I or TNM II, we did not show significant differences in prognostic value for the two widely reported 'cut off' values in either NLR or SII. The sensitivity and specificity of these scores with early TNM stage disease is therefore unlikely to be robust enough to influence treatment decisions for individual patients at presentation. However, these tools may yet have clinical value directing treatments within the BCLC algorithm for those with more advanced disease. The median NLR of 3.15, for example, distinguished significant survival differences within patients classed as either BCLC B $(n=147$ vs 87, median survival 28.9 vs 15.9 months, $P=0.013$, Kaplan-Meier) or those classed as BCLC C ( $n=248$ vs 334, median survival 9.9 vs 3.6 months, $P<0.001$, Kaplan-Meier).

Considering the individual peripheral circulating cell counts and survival analyses in the Newcastle cohort, the Hong Kong cohort or the combined cohort, it was the neutrophil count that stood out as being highly significantly and independently associated with poorer survival. Notably, in the Hong Kong cohort where the lymphocyte count was slightly lower, the lymphocyte count was independently associated with poorer survival. In this distinct geographical location the predominant underlying aetiology of HCC is chronic viral hepatitis compared with ARLD or NAFLD in Newcastle. Whether this is the cause of slightly lower lymphocytes in this geographical location is uncertain, but it is interesting as the data suggests that factors other than the cancer itself that suppress the lymphocyte count may also contribute adversely to prognosis in patients with HCC. The more consistent observation in our study, however, independent of all other factors including geographical location or underlying aetiology, was the association of poorer survival with elevated neutrophils.

Neutrophils are not only the commonest white blood cells in the circulation, they also have a shorter lifespan than the other cell types. Neutrophils typically survive $5-90 \mathrm{~h}$ in the circulation with an additional 1-2 days outside the circulation, compared with a week or so for lymphocytes and 8-9 days for platelets. Thus, we considered the possibility that these significant associations of neutrophils were enhanced relative to other cells, owing to a lesser impact of portal hypertension and hypersplenism - but the persistence of significance in the absence of CLD was against this. Neutrophils are the 'first responders' to insult or injury and it is likely that they are one of the first immune cells to come into contact with tumour cells. Our recent work in an animal model of DEN-induced HCC highlights a potential role in promoting cancer growth, as neutrophil depletion in advanced stage disease reduced tumour burden (Wilson et al, 2015). Associated mechanisms are unclear, but neutrophils are part of the spectrum of myeloid suppressor cells (MDSCs), recognised as key regulators of the tumour microenvironment, including the infiltration and phenotype of lymphocytes. The present study is on circulating cells rather than the cells in the tumour microenvironment, and how circulating neutrophil numbers reflect the tumour 
A

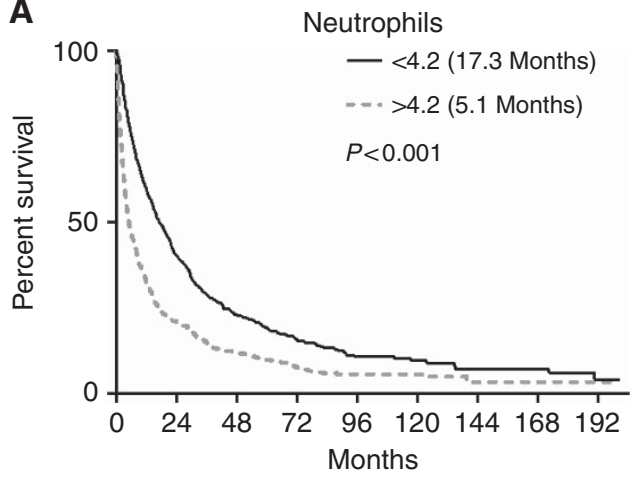

C

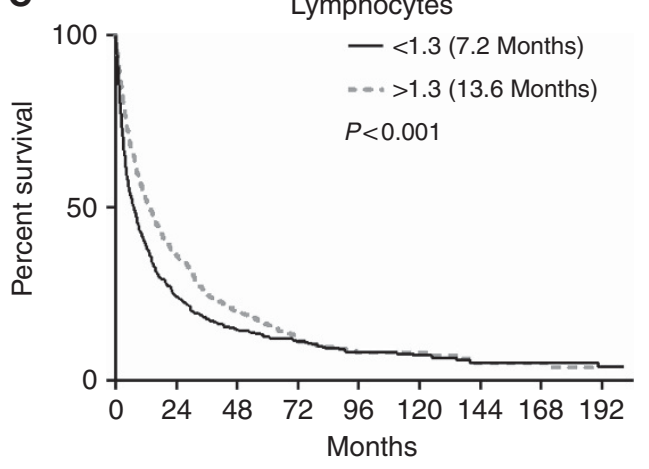

E

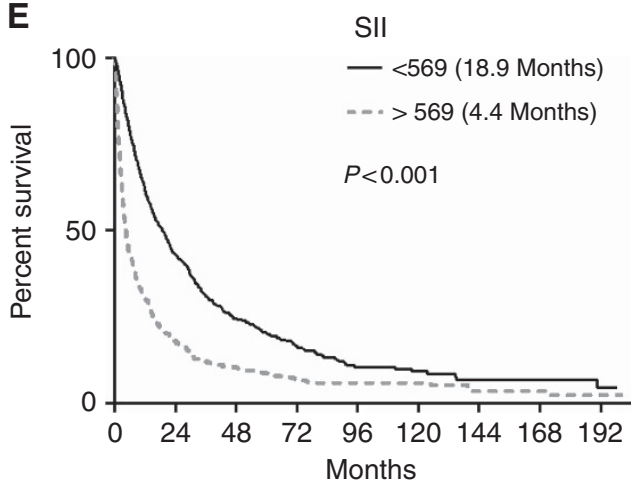

B

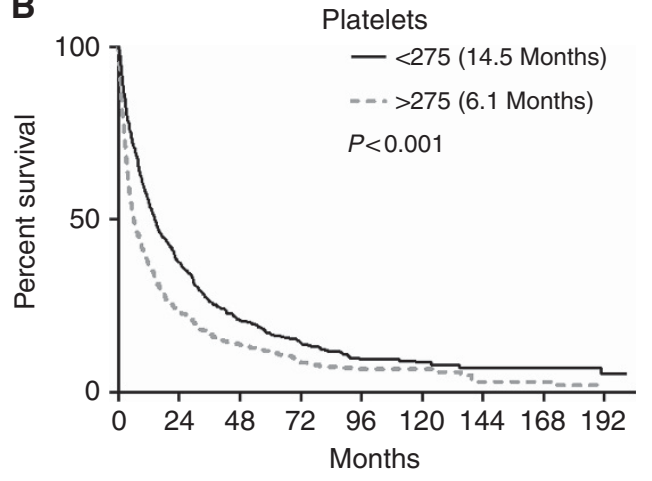

D

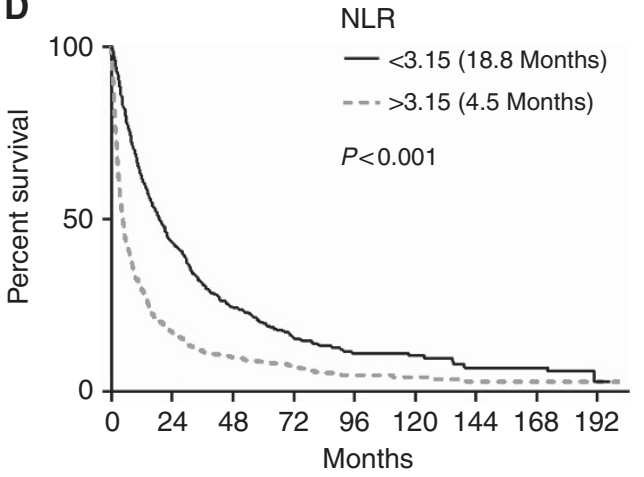

Figure 4. Inflammatory cells associations with survival. Newcastle and Hong Kong data sets combined Kaplan-Meier survival curves for neutrophils, platelets and lymphocytes, as well as NLR (neutrophil to lymphocyte ratio) and SII (Systemic Immune-Inflammation Index) are shown (A-E). In each case, two similar size groups were created based on the median value of the variable in the combined cohort. The mean value is shown on each plot, with median survival in months for those above and below the median shown in brackets. The $P$-value shown is that of the Kaplan-Meier comparisons. Univariate and multivariate Cox Regression analyses are shown in Table 2.

microenvironment is unknown. Similarly, the contribution of MDSCs to the circulating 'neutrophil' population is also unknown. In the circulation there was no apparent relationship between neutrophils and lymphocytes in the absence of CLD, although lymphocyte subtypes were not assessed. It was notable that there was a highly significant association between neutrophils and platelets in the circulation. Similar correlations between the two, with prognostic value, have been reported in other settings (Ventura et al, 1987; Watt et al, 2015). More commonly reported is the role of platelets in activating neutrophils. Immune and inflammatory responses require neutrophils to migrate through endothelial vasculature - a process dependent on their prior interaction with activated platelets (Sreeramkumar et al, 2014). In the circulation, platelets also contribute to the creation of neutrophil extracellular traps, which serve to ensnare and kill pathogens, but can inflict significant tissue damage (Kim and Jenne, 2016). In the cancer setting, similar interactions with circulating tumour cells may have roles in their elimination or metastatic implantation (Mohme et al, 2017).

In summary, by studying two consecutive series of patients totalling 1168, we have been able to validate the prognostic role of both the NLR and SII in patients with more advanced stages of HCC. Considering the individual cell types and their associations with advanced disease, we have identified that circulating neutrophils rather than lymphocytes or platelets - are significantly and independently associated with poorer prognosis and speculate that neutrophils have roles in promoting immune mediated tumour progression as well as the cancer cachexia syndrome. The relationship between circulating neutrophils - either counts or subtypes with the tumour microenvironment has yet to be established. However, we propose that neutrophil phenotype and function, including interactions or regulatory roles with platelets or lymphocytes in the presence of cancer, are worthy of study and consideration as clinically relevant therapeutic targets. 
Table 2. Predictors of survival: univariate and multivariate survival analyses

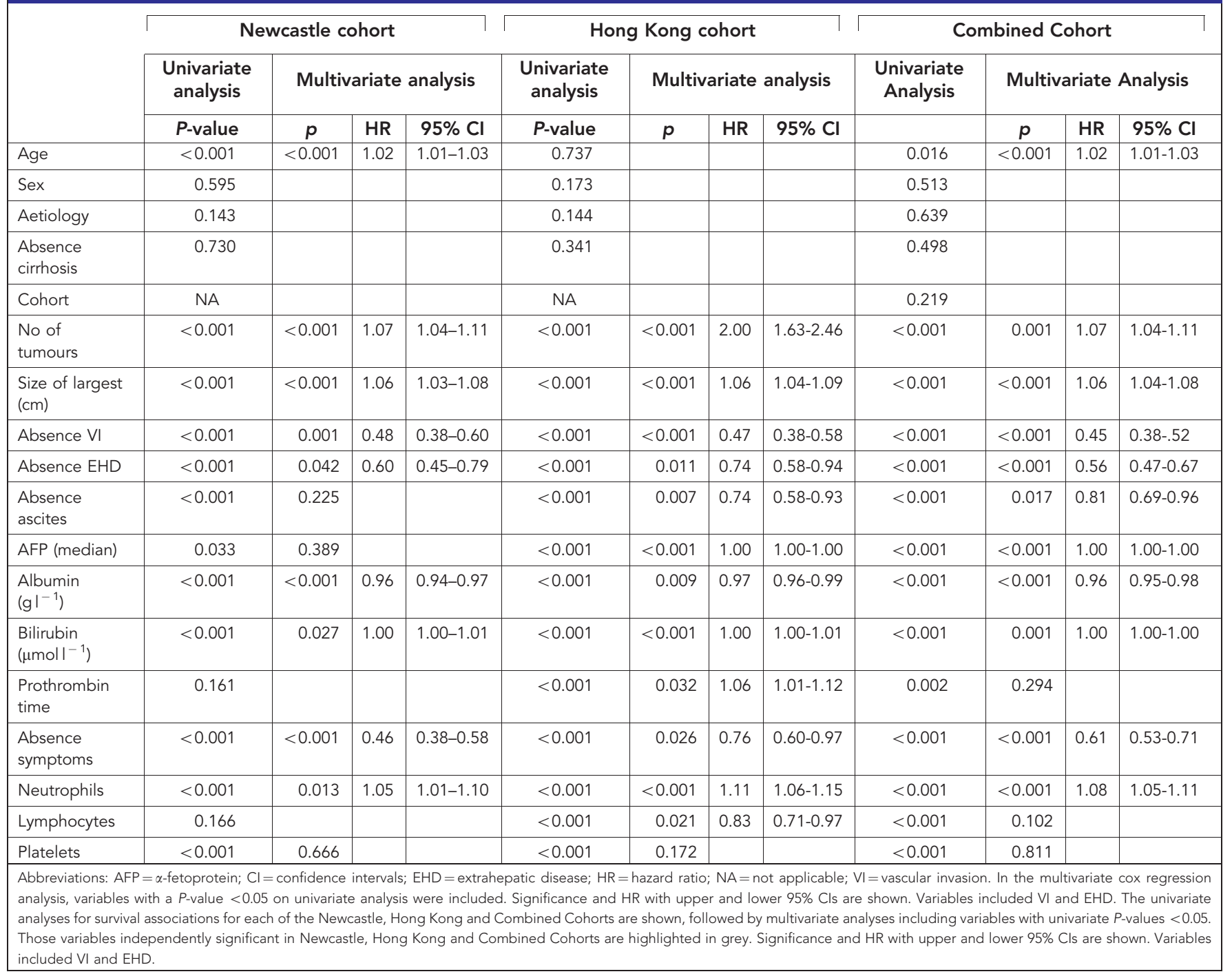

\section{ACKNOWLEDGEMENTS}

LFO was supported by a Newcastle Medical School Faculty PhD Studentship and donations from patients with HCC cared for at the Newcastle upon Tyne Hospitals NHS Foundation Trust; JM was supported by The Newcastle upon Tyne NHS Foundation Trust Research Capability Fund; CEW was supported by a Newcastle Cancer Research UK (CR UK) Centre PhD studentship. HLR, DJ, CW and DM were supported by programme grants from CR UK and the Newcastle Experimental Cancer Medicine Center. The construction of the Hong Kong cohort was supported by the Hong Kong Research Grants Council General Research Fund (number: 462013) and Terry Fox Run Hong Kong.

\section{CONFLICT OF INTEREST}

The authors of this manuscript declare the following interests or involvements that have a bearing on this paper. In particular, (1) there has been no undeclared financial support from any organisation whose finances or reputation may be affected by publication of this work; (2) there has been and is no existing or planned relationship with an organisation whose finances or reputation may be affected by the publication of the work; and
(3) there is no direct financial interest for any of the authors or their spouses, parents or children, including personal shareholdings, consultancies, patents or patent applications, whose value could be affected by the publication.

\section{REFERENCES}

Bruix J, Sherman M (2005) Management of hepatocellular carcinoma. Hepatology 42(5): 1208-1236.

Bruix J, Sherman M, Llovet JM, Beaugrand M, Lencioni R, Burroughs AK, Christensen E, Pagliaro L, Colombo M, Rodes J (2001) Clinical management of hepatocellular carcinoma. Conclusions of the Barcelona2000 EASL conference. European Association for the Study of the Liver. $J$ Hepatol 35(3): 421-430.

Cancer Research UK (2017) CR UK website. Available at: http:// www.cancerresearchuk.org/cancer-info/cancerstats/mortality/ cancerdeaths/.

Carr BI, Guerra V (2013) Hepatocellular carcinoma size: platelets, gammaglutamyl transpeptidase, and alkaline phosphatase. Oncology 85(3): 153-159.

Chan SL, Chan AW, Chan AK, Jian P, Mo F, Chan CM, Mok K, Liu C, Chong CC, Chan AT, Mok T, Yeo W (2017) Systematic evaluation of circulating inflammatory markers for hepatocellular carcinoma. Liver Int 37(2): $280-289$. 
Chan SL, Mo FK, Wong CS, Chan CM, Leung LK, Hui EP, Ma BB, Chan AT, Mok TS, Yeo W (2012) A study of circulating interleukin 10 in prognostication of unresectable hepatocellular carcinoma. Cancer 118(16): 3984-3992.

Chew V, Tow C, Teo M, Wong HL, Chan J, Gehring A, Loh M, Bolze A, Quek R, Lee VK, Lee KH, Abastado JP, Toh HC, Nardin A (2010) Inflammatory tumour microenvironment is associated with superior survival in hepatocellular carcinoma patients. J Hepatol 52(3): 370-379.

Chiba F, Soda K, Yamada S, Tokutake Y, Chohnan S, Konishi F, Rikiyama T (2014) The importance of tissue environment surrounding the tumor on the development of cancer cachexia. Int J Oncol 44(1): 177-186.

Dan J, Zhang Y, Peng Z, Huang J, Gao H, Xu L, Chen M (2013) Postoperative neutrophil-to-lymphocyte ratio change predicts survival of patients with small hepatocellular carcinoma undergoing radiofrequency ablation. PLoS One 8(3): e58184.

Dolan RD, McSorley ST, Horgan PG, Laird B, McMillan DC (2017) The role of the systemic inflammatory response in predicting outcomes in patients with advanced inoperable cancer: systematic review and meta-analysis. Crit Rev Oncol Hematol 116: 134-146.

Dyson J, Jaques B, Chattopadyhay D, Lochan R, Graham J, Das D, Aslam T, Patanwala I, Gaggar S, Cole M, Sumpter K, Stewart S, Rose J, Hudson M, Manas D, Reeves HL (2014) Hepatocellular cancer: the impact of obesity, type 2 diabetes and a multidisciplinary team. J Hepatol 60(1): 110-117.

Gomez D, Farid S, Malik HZ, Young AL, Toogood GJ, Lodge JP, Prasad KR (2008) Preoperative neutrophil-to-lymphocyte ratio as a prognostic predictor after curative resection for hepatocellular carcinoma. World J Surg 32(8): 1757-1762.

Gooden MJ, de Bock GH, Leffers N, Daemen T, Nijman HW (2011) The prognostic influence of tumour-infiltrating lymphocytes in cancer: a systematic review with meta-analysis. Br J Cancer 105(1): 93-103.

Hanahan D, Weinberg RA (2011) Hallmarks of cancer: the next generation. Cell 144(5): 646-674.

Hernandez-Gea V, Toffanin S, Friedman SL, Llovet JM (2013) Role of the microenvironment in the pathogenesis and treatment of hepatocellular carcinoma. Gastroenterology 144(3): 512-527.

Hoshida Y, Nijman SM, Kobayashi M, Chan JA, Brunet JP, Chiang DY, Villanueva A, Newell P, Ikeda K, Hashimoto M, Watanabe G, Gabriel S, Friedman SL, Kumada H, Llovet JM, Golub TR (2009) Integrative transcriptome analysis reveals common molecular subclasses of human hepatocellular carcinoma. Cancer Res 69(18): 7385-7392.

Hu B, Yang XR, Xu Y, Sun YF, Sun C, Guo W, Zhang X, Wang WM, Qiu SJ, Zhou J, Fan J (2014) Systemic immune-inflammation index predicts prognosis of patients after curative resection for hepatocellular carcinoma. Clin Cancer Res 20(23): 6212-6222.

International Agency for Research on Cancer (2014) World Cancer Report: World Health Organisation.

Kim SJ, Jenne CN (2016) Role of platelets in neutrophil extracellular trap (NET) production and tissue injury. Semin Immunol 28(6): 546-554.

Kudo M (2015) Immune checkpoint blockade in hepatocellular carcinoma. Liver Cancer 4(4): 201-207.

Limaye AR, Clark V, Soldevila-Pico C, Morelli G, Suman A, Firpi R, Nelson DR, Cabrera R (2013) Neutrophil-lymphocyte ratio predicts overall and recurrence-free survival after liver transplantation for hepatocellular carcinoma. Hepatol Res 43(7): 757-764.

Llovet JM, Fuster J, Bruix J (2004) The Barcelona approach: diagnosis, staging, and treatment of hepatocellular carcinoma. Liver Transpl 10(2 Suppl 1): S115-S120.

Llovet JM, Ricci S, Mazzaferro V, Hilgard P, Gane E, Blanc JF, de Oliveira AC, Santoro A, Raoul JL, Forner A, Schwartz M, Porta C, Zeuzem S, Bolondi L, Greten TF, Galle PR, Seitz JF, Borbath I, Haussinger D, Giannaris T, Shan M, Moscovici M, Voliotis D, Bruix J (2008) Sorafenib in advanced hepatocellular carcinoma. N Engl J Med 359(4): 378-390.

Llovet JM, Zucman-Rossi J, Pikarsky E, Sangro B, Schwartz M, Sherman M, Gores G (2016) Hepatocellular carcinoma. Nat Rev Dis Primers 2: 16018.
Mohme M, Riethdorf S, Pantel K (2017) Circulating and disseminated tumour cells - mechanisms of immune surveillance and escape. Nat Rev Clin Oncol 14(3): 155-167.

Nault JC, De Reynies A, Villanueva A, Calderaro J, Rebouissou S, Couchy G, Decaens T, Franco D, Imbeaud S, Rousseau F, Azoulay D, Saric J, Blanc JF, Balabaud C, Bioulac-Sage P, Laurent A, Laurent-Puig P, Llovet JM, Zucman-Rossi J (2013) A hepatocellular carcinoma 5-gene score associated with survival of patients after liver resection. Gastroenterology 145(1): 176-187.

Schulze K, Imbeaud S, Letouze E, Alexandrov LB, Calderaro J, Rebouissou S, Couchy G, Meiller C, Shinde J, Soysouvanh F, Calatayud AL, Pinyol R, Pelletier L, Balabaud C, Laurent A, Blanc JF, Mazzaferro V, Calvo F, Villanueva A, Nault JC, Bioulac-Sage P, Stratton MR, Llovet JM, Zucman-Rossi J (2015) Exome sequencing of hepatocellular carcinomas identifies new mutational signatures and potential therapeutic targets. Nat Genet 47(5): 505-511.

Sherman M, Bruix J (2015) Biopsy for liver cancer: how to balance research needs with evidence-based clinical practice. Hepatology 61(2): 433-437.

Sreeramkumar V, Adrover JM, Ballesteros I, Cuartero MI, Rossaint J, Bilbao I, Nacher M, Pitaval C, Radovanovic I, Fukui Y, McEver RP, Filippi MD, Lizasoain I, Ruiz-Cabello J, Zarbock A, Moro MA, Hidalgo A (2014) Neutrophils scan for activated platelets to initiate inflammation. Science 346(6214): 1234-1238.

Torbenson M, Schirmacher P (2015) Liver cancer biopsy - back to the future?! Hepatology 61(2): 431-433.

Ventura GJ, Drewinko B, Keating MJ (1987) Correlative patterns of neutrophil and platelet counts during very early remission of acute myeloblastic leukemia. Hematol Pathol 1(2): 113-116.

Villanueva A, Portela A, Sayols S, Battiston C, Hoshida Y, Mendez-Gonzalez J, Imbeaud S, Letouze E, Hernandez-Gea V, Cornella H, Pinyol R, Sole M, Fuster J, Zucman-Rossi J, Mazzaferro V, Esteller M, Llovet JM, Consortium H (2015) DNA methylation-based prognosis and epidrivers in hepatocellular carcinoma. Hepatology 61(6): 1945-1956.

Watt DG, Proctor MJ, Park JH, Horgan PG, McMillan DC (2015) The neutrophil-platelet score (NPS) predicts survival in primary operable colorectal cancer and a variety of common cancers. PLoS ONE 10(11): e0142159.

Williams R, Aspinall R, Bellis M, Camps-Walsh G, Cramp M, Dhawan A, Ferguson J, Forton D, Foster G, Gilmore I, Hickman M, Hudson M, Kelly D, Langford A, Lombard M, Longworth L, Martin N, Moriarty K, Newsome P, O'Grady J, Pryke R, Rutter H, Ryder S, Sheron N, Smith T (2014) Addressing liver disease in the UK: a blueprint for attaining excellence in health care and reducing premature mortality from lifestyle issues of excess consumption of alcohol, obesity, and viral hepatitis. Lancet 384(9958): 1953-1997.

Wilson CL, Jurk D, Fullard N, Banks P, Page A, Luli S, Elsharkawy AM, Gieling RG, Chakraborty JB, Fox C, Richardson C, Callaghan K, Blair GE, Fox N, Lagnado A, Passos JF, Moore AJ, Smith GR, Tiniakos DG, Mann J, Oakley F, Mann DA (2015) NFkappaB1 is a suppressor of neutrophil-driven hepatocellular carcinoma. Nat Commun 6: 6818 .

Xu R, Huang H, Zhang Z, Wang F-S (2014) The role of neutrophils in the development of liver diseases. Cell Mol Immunol 11(3): 224-231.

Zucman-Rossi J, Villanueva A, Nault JC, Llovet JM (2015) Genetic landscape and biomarkers of hepatocellular carcinoma. Gastroenterology 149(5): 1226-1239 e4.

This work is published under the standard license to publish agreement. After 12 months the work will become freely available and the license terms will switch to a Creative Commons AttributionNonCommercial-Share Alike 4.0 Unported License.

Supplementary Information accompanies this paper on British Journal of Cancer website (http://www.nature.com/bjc) 